\title{
PARENTING Y ESTILOS DE VIDA ADOLESCENTE SALUDABLES. EL BALANCE DE LAS AS
}

\author{
Paloma Alonso-Stuyck \\ palonsos@uic.es \\ Universidad Internacional de Cataluña \\ Instituto de Estudios Superiores de la Familia
}

Recepción Artículo: 13 marzo 2020

Admisión Evaluación: 30 marzo 2020

Informe Evaluador 1: 15 abril2020

Informe Evaluador 2: 19 abril 2020

Aprobación Publicación: 20 abril 2020

\section{RESUMEN}

Es conocida la emergencia actual de fomentar estilos de vida saludable. Éstos se conforman a instancias de patrones de conducta socioculturales e individuales. Los estilos de crianza paternos tienen un gran peso en esta tarea, especialmente en la adolescencia, momento en el que se toman las primeras decisiones de modo autónomo. Los ámbitos de decisión adolescente son ganados a medida que los padres les ceden su autoridad: se trata de un balance entre la Autoridad paterna y la Autonomía filial: el balance de las As. En este proceso es frecuente la discrepancia paternofilial sobre a quién le compete decidir. Se proponen unos estilos de crianza centrados en las dimensiones educables de la persona, capaces de ajustar expectativas entre padres y adolescentes. Esos estilos de crianza forman el clima adecuado para establecer un diálogo afectivo sobre las conductas concretas y generar canales de disciplina asertiva; la principal ventaja de estos estilos de crianza centrados en la inteligencia, voluntad y afectividad de los hijos es que se pueden adaptar a los distintos contextos socioculturales. Al fomentar la autonomía adolescente promueven la interiorización de los estilos de vida saludable a largo plazo. La adolescencia se presenta como el periodo sensible para adoptar estilos de vida saludables.

Palabras clave: estilos de vida saludable; discrepancia entre padres y adolescente; parenting; autoridad paterna; autonomía conductual adolescente; disciplina asertiva; comunicación afectiva

\section{ABSTRACT}

Parenting and healthy teenage lifestyles. The balance of the AS. The current emergency of promoting healthy lifestyles is well known. These lifestyles are shaped and driven by sociocultural and individual behavior patterns. Parenting styles carry great weight in regard to this endeavor, especially in adolescence, a time in which autonomous decision-making starts. The scope of adolescent decision-making is gained as parents cede their own authority with this process becoming a balance between parental Authority and teenage child Autonomy: balancing the As. In this process, discrepancy as to who is responsible for deciding is frequent between parent and teenage child. Parenting styles which are able to adjust expectations between parents and adolescents by 
focusing on the teachable dimensions of the person are proposed. These parenting styles create the right environment to establish affective dialogues in regard to specific behaviors and generate assertive discipline channels. The main advantage of these parenting styles rooted in the cognitive, conative and affective dimensions of children is that they can be adapted to different socio-cultural contexts. By promoting adolescent autonomy they promote the internalization of long-term healthy lifestyles. Adolescence is presented as a sensitive period in which to adopt healthy lifestyles.

Keywords: healthy lifestyles; parenting; parental authority; teenage behavioral autonomy; discrepancy between parents and teenage children; assertive discipline; affective communication

\section{ESTILOS DE VIDA ADOLESCENTE SALUDABLES}

Hace tiempo que los responsables de la salud a escala internacional alertan sobre la importancia de fomentar Estilos de Vida Saludable -EVS- (WHO) A pesar de que los programas sobre EVS se centran en los biorritmos: sueño, actividad física, dieta, etc., Ios Hábitos de Vida Saludable comprenden también aspectos psicosociales relacionados con la proactividad ante la salud emocional, o la gestión del tiempo adecuada para prevenir adicciones. Esta complejidad se manifiesta asimismo en los estudios sobre la prevención del uso de sustancias en la adolescencia, reflejando la interdependencia entre los microsistemas relacionales: vecindario, escuela, familia y grupo de pares (Calafat et al.2014; Di Luzio, Procentese \& Guillet-Descas, 2019). Efectivamente en los EVS confluyen condiciones de vida y patrones de conductas individuales y socioculturales (WHO) estrechamente relacionados con los estilos de crianza familiares. Así, las estrategias promotoras de EVS deberían conocer esos patrones para reducir riesgos en salud y fomentar el bienestar; independientemente de que se trate de una sociedad individualista 0 colectivista, horizontal o vertical, según la tipología sociofamiliar clásica (Triandis \& Gelfand, 1998).

Los Estilos de Vida se conforman a través de patrones de conducta socioculturales, en los que actualmente se perciben las siguientes paradojas (Read \& Horton, 2016; Turkle, 2014).

- Por un lado, el individualismo parece ejercer su influencia más allá de la cultura occidental, debilitando el tejido social. Al mismo tiempo la vivencia nómada postmoderna reclama más que nunca volver a las raíces estables, al sentido de pertenencia y seguridad del árbol familiar.

- En la era de la Imagen, donde las liturgias colectivas conforman identidades grupales en torno a la ficción y las fake news, emergen por contraste los movimientos minimalistas, menos es más, con realismo que desbarata la mentira social. Es decir, a pesar de la pregnancia ilusoria de los medios, se recurre a la sencillez de la relación personal y cotidiana para construir la propia identidad.

- La permanente incertidumbre relacional y laboral de la sociedad líquida junto a la falta de sostenibilidad ecológica han generado una eclosión de trastornos de ansiedad, por los que voces autorizadas instan a restaurar el equilibrio emocional enseñando a las nuevas generaciones a establecer relaciones sostenibles, amigables con uno mismo, con los demás y con el cosmos.

- Frente a la era del big-data, en un mundo dominado por los algoritmos, cuando la Inteligencia Artificial amenaza con reducir las relaciones personales a intercambios funcionales, privados de intimidad, urge revalorizar las dimensiones emocional y volitiva, respetando la rica integridad del ser humano, verdadero microcosmos natural y cultural, biológico y simbólico.

Además de estas contradicciones en los patrones de conducta socioculturales, existen diferentes vivencias y cosmovisiones. Por ejemplo, en las grandes ciudades multiétnicas de América del Norte, para fomentar EVS es necesario diseñar programas orientados a cuestiones de identidad relacionadas con ser migrantes y miembros de minorías culturales (Rousseau et al. 2014). Tanto las interacciones universales como las específicas de cada cultura influyen en los procesos de socialización relevante para la autonomía adolescente en su orientación hacia EVS (Soenens et al. 2018), aspecto que lleva a analizar el segundo aspecto conformador de los EVS, Ios patrones de conducta individuales. 
Al analizar los patrones de conducta individuales, el amplio concepto de "Estilo de Vida Familiar" relacionalmente se operativiza como el tipo peculiar de interacción entre los miembros de la familia, en interacción a su vez con los roles y expectativas sociales. En este juego de interacciones, la identidad familiar constituye la raíz de la identidad personal, de modo semejante a como el estilo relacional de los hijos se configura a partir del estilo de apego de los padres (García \& Sánchez, 2013). En el caso de que unos estilos de crianza autoritarios o permisivos hubieran promovido procesos de hostilidad o identidades confusas o evasivas, la adolescencia con el desarrollo de la autonomía constituye un punto de inflexión para restaurar el estilo relacional (Shimotomai, 2018; Kheiroddin et al.2012; Aymerich, Musitu, \& Palmero, 2018). En esa etapa evolutiva, la percepción que los hijos tienen de sus padres depende del estilo de comunicación entre ellos, aspecto relacionado con la satisfacción con la propia familia (Álvarez \& Martínez-González,2017). También las neurociencias al analizar las respuestas cerebrales apoyan que el control psicológico por parte de los padres limita el desarrollo socioemocional de los hijos y su capacidad de decisiones ponderadas (Marusak, 2018). Esta gran influencia de los estilos familiares sobre el ajuste adolescente saludable se extiende a los ámbitos clínicos. Por ejemplo, en el año posterior a un intento de suicidio, la recidiva se ve afectada por la percepción de la calidad relacional padre-hijo (Van Alphen, 2017); como también entre adolescentes con trastornos afectivos la eficacia de los programas de prevención de la dependencia a sustancias se ve reforzada por las estrategias de apoyo a la autonomía, aunque se requieran más estudios (Savard et al.2013, Spirito et al. 2015).

De la conexión entre los patrones de conducta socioculturales e individuales que conforman los EV, deriva la importancia de prevenir estilos de vida de riesgo en la adolescencia, momento en el que los hijos aprenden a tomar sus decisiones de modo autónomo. En cada familia toca equilibrar una balanza personalizada para cada hijo, que va creciendo en Autonomía al ritmo de su madurez, al tiempo que sus padres le van cediendo ámbitos de Autoridad: el balance de las As. En el origen de muchas desviaciones hacia estilos de vida adolescente de riesgo se encuentra una mala gestión de la discrepancia entre padres e hijos sobre esa cesión gradual (Rote \& Smetana, 2016). En varias revisiones se ha constatado la influencia de los estilos de crianza sobre el ajuste adolescente y el desarrollo de su autonomía (Torio \& Peña 2008; Oliva \& Parra, 2008; Gómez, 2008; Kirby, 2020). Sin embargo, faltan estudios que detallan la influencia del desarrollo de su autonomía conductual sobre la consolidación de EVS adolescentes.

\section{EVS ADOLESCENTE DESDE LA PERSPECTIVA PERSONAL DE LOS ESTILOS DE CRIANZA}

El objetivo de este trabajo es analizar esta cuestión y proponer un estilo de crianza paterna capaz de lograr el ajuste de expectativas entre padres e hijos sobre los ámbitos de autonomía adolescente, como factor promotor de los EVS. Un estilo de crianza capaz a su vez de adaptarse a las distintas culturas, para que su afectividad no se diluya por un contraste excesivo con el entorno, como propone la hipótesis de la evaporación (Hancock, 2014).

Cada familia tiende a forjar su propia dinámica relacional para promover el ajuste adolescente. Si bien se infiere que en culturas de habla inglesa parece que los estilos de crianza Autorizativos favorecen el ajuste adolescente, mientras que en otras culturas es el estilo Negligente el más adecuado (Martínez \& García, 2008; Martínez et al, 2019; García et al. 2019; Martínez et al, 2017; Capano \& Ubach, 2013; Xu et. Al, 2017; Katz et al, 2019; García, López-Fernández \& Serra, 2018; García, et al, 2018), convendría llegar a un enfoque transteórico, centrado en la persona y su comportamiento, recurriendo a postulados metateóricos capaces de aplicarse a las conductas concretas (Aroca \& Cánovas, 2012). Se requiere abrir la mente a los nuevos cambios de modo que el bienestar personal y social se mantenga a largo plazo. Steinberg (Darling \& Steinberg, 1993) proponía separar los conceptos estilo de crianza y prácticas de crianza para poder abordar procesos de socialización, como en este caso los EVS. Los estilos de crianza serían como el contexto modelador para las prácticas de crianza específicas. A la hora de promover la autonomía adolescente los estilos de crianza personales crean el humus para concretar las prácticas específicas de los ámbitos de decisión cedidos a los hijos. Decidir acertadamente en las conductas concretas es lo que conseguirá que asimilen progresivamente EVS. 
Un modo de integrar los distintos estilos de crianza desde un enfoque interactivo sistémico es centrase en las dimensiones educables de la persona, graduando en función del contexto la mayor o menor intensidad en los aspectos volitivos, emocionales o intelectivos. Para este propósito y para actualizar los cambios socioculturales está la propuesta de situar los estilos de crianza familiares en las dimensiones educables de la persona (AlonsoStuyck, 2019).

Fig. 1 Estilos de crianza situados en las dimensiones educables de la persona. Elaboración propia

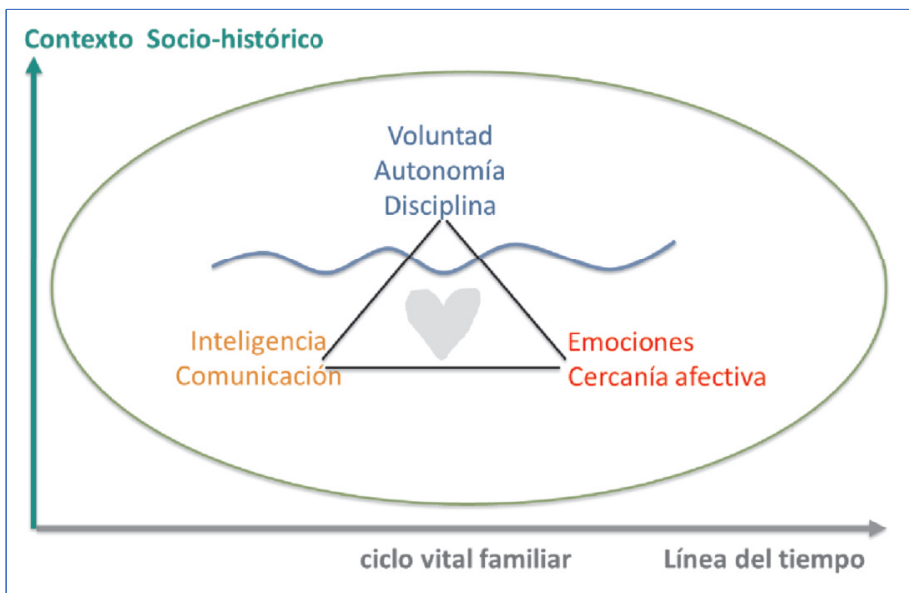

Esta manera de enfocar los estilos de crianza basada en aspectos intelectivos, emocionales y volitivos representados en la figura 1, utiliza la metáfora del iceberg, que por debajo de la línea del horizonte observable -las conductas concretas- está el sistema cognitivo-afectivo que modula a través de la argumentación propia de la comunicación y el tono afectivo de la misma, los límites familiares. Se muestra especialmente útil para crear un clima atractivo en la etapa del ciclo vital familiar con hijos adolescentes, dada la creciente influencia del grupo de amigos y del ambiente en este periodo evolutivo. Aunque mayoritariamente la pertenencia al grupo de amigos contribuye a la definición de intereses, el desarrollo de habilidades sociales y el descubrimiento de la propia identidad, no siempre su influencia resulta positiva; en ocasiones, las prácticas de socialización que caracterizan a algunos grupos se alejan de los patrones comportamentales saludables. Por este motivo se necesita invertir todos los recursos disponibles que permitan acceder a la integridad personal de los hijos.

Para superar las posibles influencias negativas del contexto, un recurso poderoso es establecer canales armónicos de disciplina que favorezcan la asertividad en las relaciones paternofiliales (García \& Gracia, 2010; Solís \& Manzanares, 2019). Se trata del aspecto volitivo de los estilos de crianza que enfoca la disciplina como un conjunto de actitudes que favorecen la autonomía adolescente y minimiza los conflictos familiares. Buscar ese delicado equilibrio entre autoritarismo y anarquía, ayuda a los hijos a autorregularse e interiorizar los EVS. Tanto si éstos deciden seguir las indicaciones parentales, como si optan por transgredirlas, los límites consensuados ayudan a los adolescentes a desarrollar la autonomía cognitiva, y por tanto asimilar unos hábitos que perduran en el tiempo. Para los padres constituye también un aprendizaje ejercer una autoridad ponderada, lograr ese punto medio entre el permisivismo y el autoritarismo, entre el dejar hacer lo que a uno le apetece 0 imponer el deber por el deber. En este clima familiar se concretarán los ámbitos de decisión que conforman la autonomía conductual adolescente en orden a su bienestar psicosocial a largo plazo (Alonso-Stuyck, 2006).

El modo de establecer los límites se verá matizado por el estilo de comunicación, oscilando entre los extremos de máxima flexibilidad y rigidez. Se concibe el estilo de comunicación como una parte de la dimensión emo- 
cional de la dinámica familiar, la más relevante para definir las relaciones paternofiliales, que suelen permanecer inalterables a lo largo de la infancia y adolescencia. El modo de establecer los límites, el estilo de comunicación incide en las dimensiones intelectiva y afectiva de los estilos de crianza y de los hijos (Serna \& Martínez, 2019; Martínez et al, 2019), tal como muestra la fig.1. En ocasiones se vincula el adecuado tono afectivo con el desarrollo del sentido del humor, tan necesario para no dramatizar las pequeñas desavenencias (Oliva, 2011); a su vez se añade el requisito de las relaciones cercanas y coherentes de los padres para el desarrollo de las habilidades prosociales en los hijos (Jiménez-lglesias, 2014). A pesar de la eclosión de programas de alfabetización emocional en los centros educativos, es sabido que su eficacia depende del estilo afectivo-relacional familiar.

En ese clima familiar que resulta de los estilos de crianza se negocian día a día los ámbitos de decisión adolescente. Atender a aspectos volitivos, intelectivos y afectivos de los hijos (disciplina, comunicación y cercanía) se combinan en cada familia a la hora de abordar la toma de decisiones en cuestiones concretas, tal como muestra la figura 2. Del modo de combinar esas dimensiones dependerá el estilo relacional de los hijos, su capacidad para establecer relaciones de confianza generando circuitos de aprendizaje positivo en entornos sociales, laborales, ecológicos, etc. desarrollando así el aspecto relacional de los EVS (Riquelme, García \& Serra, 2018). Aunque estos ámbitos específicos, y las conductas concretas que los integran, difieren en distintos ambientes culturales, lo que coincide es la discrepancia entre las expectativas de padres e hijos, el llamado fenómeno del retardo por el que los padres ven a sus hijos más pequeños de lo que éstos se consideran (Rappoport, 1986).

Fig. 2 Fenómeno del retardo, a partir de Rappoport, 1986.

\begin{tabular}{|c|c|c|}
\hline \multirow{3}{*}{$\begin{array}{l}\text { Perspectiva paterna } \\
\text { Perspectiva } \\
\text { adolescente }\end{array}$} & $\begin{array}{c}\longrightarrow \text { Ámbito de decisión } \\
\text { de los padres }\end{array}$ & $\begin{array}{c}\text { Ámbito de decisión } \\
\text { de los hijos }\end{array}$ \\
\hline & $\begin{array}{l}\text { Ámbito de } \\
\text { decisión de } \\
\text { los padres }\end{array}$ & $\begin{array}{l}\text { Ámbito de decisión } \\
\text { de los hijos }\end{array}$ \\
\hline & 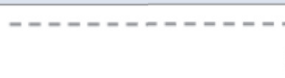 & VIDA \\
\hline
\end{tabular}

No deja de ser una paradoja el que, si bien padres y adolescentes desean de manera global lo mismo: que los hijos lleguen a valerse por sí mismos y aprendan a decidir sin la recurrir a sus padres, cuando llega el momento de que los hijos demandan mayor autonomía los padres piensen que no están preparados. Esta discrepancia requiere un ajuste de expectativas amistoso, que promueva un diálogo cercano y realista (Rote \& Smetana, 2016). Al revisar las investigaciones sobre funcionamiento familiar en la adolescencia resaltan la constante discrepancias entre padres e hijos: al abordar las dinámicas de relación -p. ej. conflicto (Özdemir, Vazsonyi \& Çok, 2017)-, la supervisión del comportamiento filial por parte de los padres -p. ej. monitoreo (De los Reyes \& Ohannessian, 2019)-, o las percepciones de los hijos (p. ej. legitimidad de la autoridad de los padres (GonzalesBacken, 2019; Yeung., Tsang \& Chen, 2019).

En los distintos contextos culturales conviene estudiar los valores y actitudes de los adolescentes que generan esa discrepancia (Rousseau, 2015; Bi, 2018); por ejemplo, todavía no está claro el efecto de las brechas de aculturación entre padres e hijos, más acusadas en familias migrantes (Wang-Schweig \& Miller, 2018). En todo caso la resolución del conflicto parece proceder de la falta de ajuste de expectativas entre padres e hijos sobre sobre quién debe decidir qué (Spirito et al, 2015; Alonso-Stuyck, 2006). Para resolver cualquier problema hay que empezar por definirlo bien, por tanto, el primer paso sería establecer a que categoría pertenece para padres e hijos cada tema de discusión, que de manera clásica se han dividido en 3 áreas (figura 3). La Autonomía con- 
ductual de los hijos va creciendo como por un plano inclinado en una trayectoria inversa a la cesión de Autoridad de los padres sobre esos mismos temas. La doble dirección de la flecha indica que a medida que va aumentando la esfera de decisión de los hijos, disminuyen correlativamente los ámbitos de decisión de los padres.

Figura 3. Inversión entre los ámbitos de decisión de hijos y padres. Elaboración propia

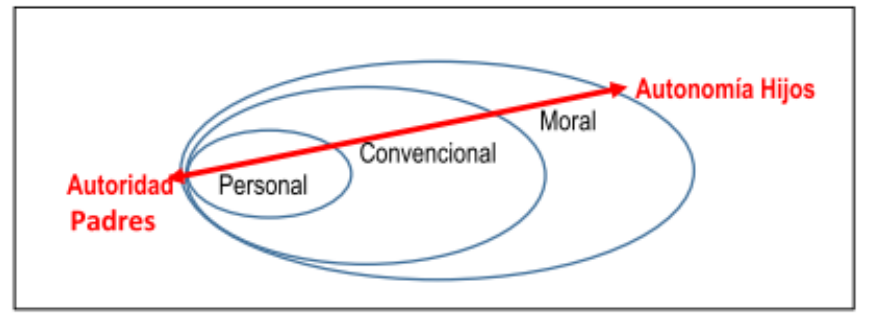

En general tanto padres como hijos suelen aceptar que los asuntos morales y socio-convencionales los decidan los padres, pero con frecuencia ambos sitúan los mismos temas en distintas categorías. Algunos cuestionarios ofrecen una relación de estas conductas (Bosma \& Jackson, 1996) que podrían definirse y agruparse del siguiente modo:

\ Moral: incluye asuntos que comprometen la conciencia, porque rozan la justicia, los derechos, o el bienestar físico y psicológico de las personas. Con qué amigos se sale; cuánto alcohol consumir; dónde ir al salir; si fumar o no fumar; qué hacer y no con relación a la sexualidad, participar en actividades religiosas.

- Socioconvencional: cuando se refieren a la praxis del orden grupal, es decir a las normas establecidas para asegurar la convivencia social y familiar: Ayudar en las tareas de casa; con qué frecuencia visitar a los familiares y parientes; hora de vuelta a casa por la noche; cómo comportarse en las comidas; uso del lenguaje y manera de hablar; hora de irse a dormir; qué ropa llevar; aspecto, estilo de peinado, apariencia general.

- Privado/prudencial: los temas relacionados con el proyecto personal. Cómo gastar el dinero asignado; en qué deportes participar, qué aficiones o pasatiempos realizar, tiempo dedicado a los deberes escolares, frecuencia del baño o ducha, dulces y chocolates que se toma, asuntos personales o privados.

Es frecuente que el grado de discrepancia sea significativo en las tres categorías, menos en algunas conductas muy concretas, como tareas de casa, visitas $(\boldsymbol{\bullet}) 0$ dinero $(\bullet)$, mientras que se mantiene la discrepancia en todos los temas morales $(\mathbf{\Delta})$.

Este dato refleja la necesidad de mayor diálogo entre padres e hijos, especialmente en los temas que dan sentido a sus vidas encaminándolos a EVS. Se trata de aprender a establecer una negociación reflexiva en la que todas las partes salgan con la sensación de haber ganado. El acierto se fundamenta en que la Autonomía adolescente tienda a la interdependencia y que los padres no se dejen solos a los hijos en su cesión gradual de Autoridad. Frente a esta negociación, la cultura de la imagen parece alentar los enfrentamientos irreflexivos donde las emociones arteramente manejadas son capaces de conquistar o destruir cualquier logro. Si el conflicto entre padres y adolescentes puede considerarse un rito de transición adolescente (Alonso-Stuyck \& Aliaga, 2017), resulta decisivo el modo de afrontarlo para que no devengan en problemas de ajuste para los adolescentes (Ohannessian, 2012). Los beneficios de mantener en la familia espacios de diálogo, a pesar de las desavenencias propias del periodo adolescente, se traducen tanto en el desarrollo positivo de la personalidad como en estilos de vida saludables (Álvarez, 2017).

\section{DISCUSIÓN Y CONCLUSIONES}

Se puede considerar la adolescencia un periodo crítico para la configuración de los EVS a través de las tendencias que va acrisolado la recién estrenada autonomía conductual, de modo semejante a como la infancia lo 
es para la personalidad a instancias de las vivencias afectivas; no significa que la persona quede ya determinada por ello, sino en cierta medida condicionada.

Los estilos de crianza paternos centrados en las dimensiones educables de la persona: inteligencia, voluntad y afectividad, se presentan como el clima adecuado para fomentar una autonomía adolescente acorde a los EVS. Esos estilos de crianza se caracterizan por un diálogo afectivo capaz de acercar las expectativas de padres e hijos sobre el significado de las conductas específicas - pautas de crianza- que conforman los ámbitos de decisión moral, socioconvencional y personal. Establecer esos cauces de disciplina asertiva, significa balancear las As, es decir, conjugar una Autoridad paterna cercana, con una Autonomía adolescente interdependiente, capaz de mantener relaciones personales sostenibles.

Los cuestionarios que desgranan las conductas que con más frecuencia son motivo de discrepancia entre padres y adolescentes sobre quien debe decidir qué, se presentan como recursos poderosos para ensayar ese diálogo afectivo, del que derivan los canales de disciplina asertiva. La normalización del conflicto junto al recurso al diálogo socrático, anima a los hijos a expresar su opinión y genera la confianza. Cada familia discernirá si el contenido de las discusiones se centra únicamente en asuntos cotidianos evitando temas más profundos, y si éstos no se abordan por falta de tiempo, por horarios laborales excesivos que limitan la convivencia, momento esencial del dinamismo formativo.

La flexibilidad generadora de alternativas al tomar decisiones tendría que estar presente también desde la infancia. A la apertura paciente a las propuestas de los hijos se une la experiencia de la responsabilidad, dejarles afrontar las consecuencias de sus actos. Un equilibrio alejado tanto del intervencionismo excesivo para evitar que Ios hijos se equivoquen -sobreprotección-, como de la ausencia de normas -permisivismo-. La mirada apreciativa que propicia el acercamiento confiado a los padres podría constituir la clave: la escucha aceptante focalizada positivamente en las propuestas de los hijos sería un factor decisivo para lograr la adhesión esperada a os EVS.

Estas características del funcionamiento familiar conformarían una metodología de ajuste de expectativas entre padres y adolescentes apoyada en el balance Autoridad-Autonomía dirigida a convertir este periodo, aparentemente difícil, en una oportunidad de crecimiento familiar. A los padres les compete el arte de transmitir su patrimonio experiencial (no solo biológico o económico), afinando la conciencia de sus hijos, conociéndolos bien, para despertar en ellos el amor a la vida y promover que se abran a ella con libertad; y a los hijos asumir sus determinantes biológicos y sociales sin plegarse a ellos: dentro del amplio margen marcado por la genética y la educación están llamados a construir su identidad madura.

Materiales suplementarios: Los siguientes están disponibles en línea en www.mdpi.com/xxx/s1, Fig. 1 Estilos de crianza situados en las dimensiones educables de la persona. Fig. 2 Fenómeno del retardo. Figura 3. Inversión entre los ámbitos de decisión de hijos y padres.

\section{REFERENCIAS BIBLIOGRÁFICAS}

Alonso-Stuyck, P. (2006). Discrepancia padres/hijos en percepción del funcionamiento familiar y desarrollo de la autonomía adolescente. PhD thesis, Universidad de Valencia, España.

Alonso-Stuyck, P. (2019). Which Parenting Style Encourages Healthy Lifestyles in Teenage Children? Proposal for a Model of Integrative Parenting Styles. Int. J. Environ. Res. Public Health 16, (11) 2057.

Alonso-Stuyck, P. \& Aliaga, F. (2017). Demanda de autonomía en la relación entre los adolescentes y sus padres. ESE 33, 77-101.

Álvarez, A. (2017). Is Family Life Relevant to Social Justice? Ideas y valores 163(5), 81-121.

Álvarez, L. \& Martínez-González, R. A. (2017). Review of the Partnership between School and Family: A Shared Responsibility. In Factors affecting academic performance; González-Pienda, A.,

Bernardo, J. C., Nuñez C., Eds; New York: Nova Science Publishers, 121-140.

Aroca, C. \& Cánovas, P. (2012). Los estilos educativos parentales desde los modelos interactivos y de construcción conjunta: Revisión de las investigaciones. Teoría de la Educación 24, 149-176. 
Aymerich, M., Musitu, G., \& Palmero, F. (2018). Family Socialization Styles and Hostility in the Adolescent Population. Sustainability 10 (9). doi:10.3390/su10092962

Bi, X., Yang, Y., Li, H., Wang, M., Zhang, W. \& Deater-Deckard, K. (2018). Parenting styles and parent-adolescent relationships: The mediating roles of behavioral autonomy and parental authority. Frontiers in Psychology 9. https://doi.org/10.3389/fpsyg.2018.02187

Bosma, H. \& Jackson, S. E. (1996). Who was the final say? Decisions on adolescent behavior within the family. Journal of adolescence 19, 277-291.

Calafat, A., García, F., Juan, M., Becoña, E. \& Fernández-Hermida, JR. (2014). Which parenting style is more protective against adolescent substance use? Evidence within the European context. Drug and Alcohol Dependence 138, 185-192. doi: 10.1016 / j.drugalcdep.2014.02.705.

Capano, A. \& Ubach, A. (2013). Parenting styles, positive parenting and parent's formation. Cienc. Psicol 7 (1)

Darling, N. \& Steinberg, L. (1993). Parenting style as context: An integrative model. Psychological Bulletin 113(3), 487-496. https://doi.org/10.1037/0033-2909.113.3.487

De los Reyes, A. \& Ohannessian, C. (2019). Discrepancies between Adolescent and Parent Reports about Family Relationships. Child Development Perspectives 12 . D0I: 10.1111/cdep.12306

Di Luzio, S., Procentese, F. \& Guillet-Descas, E. (2019). Physical Activity in Adolescence and Substance Use: Factors of Interdependence between Local Community and Relational Micro-Systems. Journal of Child \& Adolescent Substance Abuse 28(2) 119-126. http://dx.doi.org/10.1080/1067828X.2019.1623144

García, F. \& Gracia, E. (2010). ¿Qué estilo de socialización parental es el idóneo en España? Un estudio con niños y adolescentes de 10 a 14 años. Infancia y Aprendizaje 33 (3), 365-384.

García, F., Serra, E., García, 0. F., Martinez, I., \& Cruise, E. (2019). A third emerging stage for the current digital society? Optimal parenting styles in Spain, the United States, Germany, and Brazil. ljerph 16(13), 2333.

García, M.J. \& Sánchez, R.F. (2013). La familia occidental en el siglo XXI: una perspectiva comparada. ESE 25, $31-47$.

García, 0. F., López-Fernández, 0. \& Serra, E. (2018). Raising Spanish Children with an Antisocial Tendency: Do We Know What the Optimal Parenting Style Is? Journal of Interpersonal Violence 1 -28. D0I: $10.1177 / 0886260518818426$

García, 0.F., Serra, E. Zacarés, J.J. (2018). García, F. Parenting Styles and Short- and Long-term Socialization Outcomes: A Study among Spanish Adolescents and Older Adults. Psychosocial Intervention 27(3), 153161. http://journals.copmadrid.org/pi

Gómez, E. (2008). Adolescencia y familia: revisión de la relación y la comunicación como factores de riesgo 0 protección. Revista Intercontinental de Psicología y Educación 10(2), 105-122.

Gonzales-Backen, M. (2019). Parenting Practices and Adjustment Profiles among Latino Youth in Rural Areas of the United States. Soc. Sci 8 (6), 184; https://doi.org/10.3390/socsci8060184

Hancock, D. (2014). Consequences of Parenting on Adolescent Outcomes. Societies 4, 506-531; doi:10.3390/soc4030506

Jiménez-Iglesias, A.; Moreno, C.; García-Moya, I. \& López, F. (2014). The family relationships in the adolescents' voice. Journal of Child and Adolescent Psychology 5(2), 11-30.

Katz, I., Lemish, D., Cohen, R. \& Arden, A. (2019). When parents are inconsistent: Parenting style and adolescents' involvement in cyberbullying. Journal of Adolescence 74, 1-12.

Kheiroddin, J.B; Esmaeili, B; Gholamzadeh, M. \& Mohammadpoor, W. (2012). The relationship between parenting styles and identity styles in adolescent. Journal of Psychology 16(2), 161-175.

Kirby, J. N. (2020). Nurturing Family Environments for Children: Compassion-Focused Parenting as a Form of Parenting Intervention. Education Sciences, 10(1), 3.

Martínez, I et al. (2019). Researching parental socialization styles across three cultural contexts: Scale ESPA29 bi-dimensional validity in Spain, Portugal, and Brazil. ljerph 16(2), 197. 
Martínez, I., \& García, J. F. (2008). Internalization of values and self-esteem among Brazilian teenagers from authoritative, indulgent, authoritarian, and neglectful homes. Adolescence-San diego 43(169), 13

Martínez, I., Cruise, E., García, Ó. F., \& Murgui, S. (2017). English validation of the parental socialization scaleESPA29. Frontiers in psychology, 8, 865.

Martínez, I., Murgui, S., García, O. F., \& García, F. (2019). Parenting in the digital era: Protective and risk parenting styles for traditional bullying and cyberbullying victimization. Computers in Human Behavior 90, 84-92.

Marusak, H., Thomason, M., Sala Hamrick, K., Crespo, L. \& Rabinak, C. (2018). What's parenting got to do with it: emotional autonomy and brain and behavioral responses to emotional conflict in children and adolescents. Developmental science 21, 4. https://doi.org/10.1111/desc.12605

Ohannessian, C. (2012). Parental Problem Drinking and Adolescent Psychosocial Adjustment: The Mediating Role of Adolescent-Parent Communication. Journal of Research on Adolescence 22(3):498-511. D0I: 10.1111/j.1532-7795.2012.00791.x

Oliva, A. (2011). Apego en la adolescencia. Acción Psicológica. D0l: http://dx.doi.org/10.5944/ap.8.2.190

Oliva, A., Parra, A. \& Arranz, E. (2008). Estilos relacionales parentales y ajuste adolescente. Infancia y Aprendizaje, 31(1), 93-106.

Özdemir Y., Vazsonyi AT. \& Çok F. (2017). Parenting processes, self-esteem, and aggression: A mediation model. EUR. J. Dev. Psychol 14: 509-532. https://doi.org/10.1080/17405629.2016.1240674

Rappoport, L. (1986). La personalidad desde los 13 a los 15 años. Barcelona: Paidós.

Read J.C., \& Horton M. (2016). Future Directions for Quality Teen Cl Research. In Perspectives on HCl Research with Teenagers. Human-Computer Interaction Series. Little L., Fitton D., Bell B.,

Toth N, Eds. Springer. https://doi.org/10.1007/978-3-319-33450-9_11

Riquelme, M., García, 0.F. \& Serra, E. (2018). Desajuste psicosocial en la adolescencia: socialización parental, autoestima y uso de sustancias. Anales de psicología 34 (3), 536-544.

Rote, W.M. \& Smetana, J.G. (2016). Patterns and predictors of mother-adolescent discrepancies across family constructs. Journal of Youth and Adolescence, 10, 2064-2079.

Rousseau, C., Gauthier, M.F., Lacroix, L., Alain, N., Benoit, M., Moran, A., Rojas, M.V. \& Bourassa, D. (2015). Playing with identities and transforming shared realities: Drama therapy workshops for adolescent immigrants and refugees. The Arts in Psychotherapy 32(1), 13-27.

Savard, A., Joussemet, M. \& Emond Pelletier, J. et al. (2013). The benefits of autonomy support for adolescents with severe emotional and behavioral problems. Motiv Emot 37: 688. https://doi.org/10.1007/s11031-0139351-8

Serna, C., \& Martínez, I. (2019). Parental Involvement as a Protective Factor in School Adjustment among Retained and Promoted Secondary Students. Sustainability 11(24), 7080.

Shimotomai, A. (2018). Parental social power, co-parenting, and child attachment: early to late japanese adolescence transitions. Current Psychology https://doi.org/10.1007/s12144-018-9811-0

Soenens, B., Park S-Y., Mabbe, E., Vansteenkiste M., Chen, B., Van Petegem, S. \& Brenning K. (2018). The Moderating Role of Vertical Collectivism in South-Korean Adolescents' Perceptions of and Responses to Autonomy-Supportive and Controlling Parenting. Front. Psycho/ https://doi.org/10.3389/fpsyg.2018.01080

Solís G.L. \& Manzanares, E. (2019). Parental Psychological Control and Internalized and Externalized Problems of Adolescents in Metropolitan Lima. Revista colombiana de psicología 28 (1), 29-47.

Spirito, A., Hernández, L., Cancilliere, MK., Graves, H. \& Barnett, N. (2015). Improving Parenting and ParentAdolescent Communication to Delay or Prevent the Onset of Alcohol and Drug Use in Young Adolescents with Emotional/Behavioral Disorders: A Pilot Trial. Journal of Child \& Adolescent Substance Abuse 24:5, 308-322. https://doi.org/10.1080/1067828X.2013.829013

Torio, S., Peña, J.V. \& Rodríguez, M.C. (2008). Estilos educativos parentales. Revisión bibliográfica y reformulación teórica. Teoría de la Educación 20, 151-178. 
Triandis, H. C. \& Gelfand, M. J. (1998). Converging measurement of horizontal and vertical individualism and collectivism. Journal of Personality and Social Psychology 74, 118-128.

Turkle, S. (2013). Alone together: Why we expect more from technology and less from each other. Perseus Books.

Van Alphen,N.R., Stewart, J.G., Esposito, E.C. Pridgen, B. Gold, J. \& Auerbach, R.P. (2017). Predictors of Rehospitalization for Depressed Adolescents Admitted to Acute Psychiatric Treatment? J Clin Psychiatry 78(5) 592-598. doi: 10.4088/JCP.15m10326

Wang-Schweig, M. \& Miller, BA. (2018). Youth Adolescence Correction to: Examining the Interdependence of Parent-adolescent Acculturation Gaps on Acculturation-based Conflict: Using the Actor-Partner Interdependence Model. Journal of Youth and Adolescence https://doi.org/10.1007/s10964-018-0948-9

WHO. Health Promotion Glossary. Available online: https://apps.who.int/iris/handle/10665/67246 (accessed on 1 March 2020)

WHO. Life-Styles and Health. Available online: http://apps.who.int/iris/bitstream/handle/10665/85649/ Official_record50_eng.pdf?sequence=1\&isAllowed=y (accessed on 1 March 2020)

Xu. J., Ni, S., Ran, M. \& Zhang, C. (2017). The Relationship between Parenting Styles and Adolescents' Social Anxiety in Migrant Families: A Study in Guangdong, China. Frontiers in Psychology. D0I: 10.3389/fpsyg.2017.00626

Yeung, WK., Tsang, E. \& Chen, H. (2019). Parental Socialization and Development of Chinese Youths: A Multivariate and Comparative Approach. Int. J. Environ. Res. Public Health 16(10), 1730; https://doi.org/10.3390/ijerph16101730 